\title{
Assessing the Nature of Job Satisfaction Level: A Study on Private Bank Employees in Bangladesh
}

\author{
Md. Najmul Hasan ${ }^{1}$, Shazia Afrin ${ }^{2}$, Mostafijur Rahman ${ }^{3 *}$, Alim Al Ayub Ahmed ${ }^{4}$
}

${ }^{1}$ Assistant Professor, Department of Business Administration, First Capital University of Bangladesh, Chuadanga, BANGLADESH

${ }^{2,3}$ Lecturer, Department of Business Administration, First Capital University of Bangladesh, Chuadanga, BANGLADESH

${ }^{4}$ Professor (Visiting), School of Accounting, Jiujiang University, Jiujiang, Jiangxi, CHINA

*Corresponding Contact:

Email: mostafizrumgt@gmail.com

Manuscript Received: 27 June 2020 - Revised: 28 Aug $2020 \quad$ - Accepted: 15 Sept 2020

\begin{abstract}
The purpose of this study is to assess the nature of the job satisfaction level of private bank employees in Bangladesh. It used a semi-structured questionnaire which contains both pre-coded and open-ended questions. All questions were rated with the Likert 5point scale. A Chi-square test was used to assess the relationship between independent and dependent variables. In this study, a significant inter-class relationship was observed between demographic characteristics namely sex, age, designation, salary, and family member, marital status, working environment, service period and family income, and job satisfaction indicators which are, participation in decision making, training facilities, autonomy in work, gender discrimination, working hour, chance of promotion, increase knowledge \& capacity, the practice of $\mathrm{MBO}$ and surprisingly availability of tools and resources had no significant relation with any demographic factors. Employees who work as an officer $(93.3 \%, \mathrm{p}<.001)$ and withdraw salary 25,001 $35,000(88.2 \%, \mathrm{p}<.000)$ cannot participate in decision making. But who works in the participative environment $(83.9 \%, \mathrm{p}<.000)$ get a proper training facility. Employees with $<30$-year age $(83.3 \%, \mathrm{p}<.016)$ cannot practice autonomy. Unmarried workers $(53.8 \%, \mathrm{p}<.006)$ face gender discrimination. Employees who work in an Autonomous environment $(76.0 \%, \mathrm{p}<.00001)$ do not get enough working hours. Employees with 30 35 -year age $(80.8 \%), \mathrm{p}<.002)$ do not get a proper promotion. Male $(98.0 \%, \mathrm{p}<.001)$ report the organization increases its capacity and knowledge. Employees work in an autonomous environment $(88.0 \%, \mathrm{p}<.002)$ report that organization practices MBO. This study also shows that female employees are more satisfied than males. The overall situation can be improved by guaranteeing employees participation in decision making, regular training, providing appropriate increments and promotion, redesigning working hours and environment, and removing gender discrimination.
\end{abstract}

Key Words: Job satisfaction, Chi-square test, demographic characteristics, Bank employees, Bangladesh 


\section{INTRODUCTION}

Employees are most significant resources of any business and it is commonly recognized (Farooqui, \& Nagendra, 2014). Employees' contribution to enterprise largely depends on their satisfaction (Hassan et al., 2020). Satisfaction ensures employees happiness and full enthusiasm and it turned them to prime resource any organization including bank (Khan 2015). Job satisfaction has a significant role not only in individual success but also in the economic development of a nation (Faragher et al., 2005). It also has vital effect on employee's turnover intension, absenteeism, and job performance. On contrary, Unsatisfaction has adverse effect on mental health and cause depression (Balcazar, 2020). As Employees are the key sources of competitive advantage so business organization has to find out the sources of satisfaction to confirm it (Agarwala 2011; Haque \& Ahmed, 2016).

Job satisfaction does not merely depend on designation rather on organization culture, good relation with different parties, management pattern, social and physical environment (Bayona et al., 2020). Vroom 1964 narrated Employees' happiness to job means job satisfaction (Huning et al., 2020). It is a feeling aroused in response to a comparison between expected and actual outcomes on the work (Moortezagholli, 2020). More simply, job satisfaction is the degree to which employees love their profession (Hakim, 2015).

Organizations who have more satisfied employees are more effective and productive than those with more dissatisfied employees (Ahmed, 2020). The degree of satisfaction is determined by the ratio between what we have and what we want. When workers are free to decide how to develop and organize their work, they often feel more satisfied and committed to their job (Islam, 2016). High employee satisfaction will strengthen and support the workforce, make employees more creative and innovative and produce superior performance and productivity in optimal time, diminish absenteeism and turnover intention, reduce accident, stress, and work performance as a whole improves (Budría \& Baleix, 2020; Asadullah et al., 2019; Rahman, 2020), thus it increases productivity and profits (Farrell and Stamm, 1988).

On the other hand, job dissatisfaction has adverse consequence on employee's performance (Shaikh et al., 2019) like as, it creates economic pressure which lead unemployment and inflation (Kumar, 2016), increases employees' turnover rate (Chaulagain \& Khadka, 2012). It cut employees performance and increases cost (Clark, 1997) and employees not only feel unsafe (Hoboubi et al., 2017) but also unhappy and frustrated at their jobs (James, 2020).

Several studies have been conducted regarding job satisfaction in Bangladesh and worldwide where a majority of these studies measured either factor related to job satisfaction or level of job satisfaction. Islam et al. (2012) stated that coordination and leave facility, reward \& future opportunities, the vision of the company, work process, and health and insurance policy factors have a significant influence on job satisfaction. Huda et al. (2011) showed that garment workers are unhappy with their working environment and about pay package.

Ireri K (2016) found income, job security, and job autonomy were the main predictors of Kenyan journalist's job satisfaction. On the other hand, working environment and administration, supervisors \& working hours and security of income are mainly responsible for employees' job dissatisfaction (Talukder et al., 2014). Public bank 
employees were more satisfied than private and pay, job safety, acknowledgement, work itself influences their satisfaction (Weerasinghe et al., 2017).

As satisfaction of employees to job determines performance, so organization performance is strongly linked with job satisfaction (Bakotic, 2016). Employee satisfaction is positively correlated with market performance and organization performance which can measured by earnings per share and production, profit, employee turnover, employee accidents, and customer satisfaction respectively (Evans \& Jack, 2003). It is also optimistically associated with financial indicators which are return on equity, revenue per employee, per employee labor costs and index BEX (Earnings before taxes and interest) (Bakotic, 2016). Frederick Herzberg, in his renowned two factor theory motivation factors (achievement, recognition advancement, responsibility, and work itself) and hygienic factors (company policy, salary, working conditions, and interpersonal relations), revealed that insufficiency of hygienic factors or extrinsic reward cause employee's dissatisfaction while availability of motivation factors or intrinsic reward ensures job satisfaction (Herzberg et al., 1959).

Lower level of job satisfaction was found among middle age and married employees, whom work as an officer, got average salary and work longer period of time in autonomous environment (Rahman et al., 2017). Employees commitment to organization is also include by their satisfaction (Falkenburg and Schyns, 2007). Antoncic and Antoncic (2011) showed any work has four dimension namely general satisfaction with work; employee relationships; remuneration, benefits and organizational culture; and employee loyalty. These dimensions are positively associated with employee satisfaction which foster overall growth of business.

However, to best of our knowledge, there is hardly any study which assesses the nature of job satisfaction level of private bank employees in Bangladesh. So, the objective of this study is assessing the nature of the job satisfaction level of private bank employees in Bangladesh. 


\section{Methodology}

There are 7 private banks are operating in Chuadanga and all the 7 banks have been selected for this study. We included Mercantile Bank Ltd., DBB Ltd., Islami Bank Bangladesh Ltd., SIBL, First Security Islami Bank Ltd., BRAC Bank Ltd. and UCB Ltd banks in our study.

The cross-sectional study sample consisted of 56(male 50 and female 6) bank employee's respondents. They were interviewed from $1^{\text {st }}$ January to $26^{\text {th }}$ February 2020.

A semi-structured questionnaire was developed using job satisfaction forces to which the respondents were asked to react using a three Bipolar scale ranging which was (i) Yes (ii) No and (iii) No comment.

\section{Sample Size}

This study used Yamane (1967) formula to calculate sample size (50). Yamane (1967) provided a simplified formula to calculate sample size.

Equation is-

$$
\mathrm{n}=\frac{N}{1+N\left(e^{2}\right)}
$$

Where

$\mathrm{n}=$ is the sample size

$\mathrm{N}=$ is the population

$1=$ is a constant

$e^{2}=$ is the estimated standard error which is $5 \%$ for $95 \%$ confidence level

$$
\mathrm{n}=\frac{66}{1+66\left(0.05^{2}\right)}=56.65 / 56
$$

Dependent variables: We use several independent variables such as Gender, Age, Religion, Education, Designation, Salary, Family Member, Marital status, Geographic Location, Family income, Service period and the Working environment in this study.

Independent variables: To assess the nature of the job satisfaction level nine (9) dependent variables have been used which are categorized decoratively. The dependent variables are 'Participation in decision', 'Availability of Tools and Resources', 'Training facilities', 'Autonomy in work', 'Gender discrimination', 'Enough working hour', chance promotion', 'increase knowledge \& Capacity', and 'Practice of Manage by Objective (MBO)'.

\section{Statistical analysis}

SPSS 20.0 and Excel have been used to process and analyze the data. Both dependent and independent variable are analyzed by using Chi-square test as Chi-square test is very useful to show the relationship between two variables. It has been used to test the statistical significance of the parameters at $5 \%$ level.

The written consent has been taken from the respondents. This study is approved by the research authority of the First Capital University of Bangladesh. 


\section{RESULT}

Table 1 shows employee's responses toward the questions in number and percentage. $51.8 \%$ employees say they have available tools and resources, $67.9 \%$ cannot participate in decision making, more than half of respondents $(57.1 \%)$ have proper training facility, $66.1 \%$ cannot practice autonomy in their working area, $1 / 4$ respondents face gender discrimination, almost half $(51.8 \%)$ get enough hour to work, $55.4 \%$ do not get proper promotion, around all respondents (96.6\%) say their knowledge and capacity is increasing and $66.1 \%$ give positive report about $\mathrm{MBO}$.

Table 1: Showed employees responses toward the questions in number and percentage

\begin{tabular}{|c|c|c|}
\hline Variables & Number & Percentage $(\%)$ \\
\hline \multicolumn{3}{|c|}{ Tools and Resources } \\
\hline No & 27 & 48.2 \\
\hline Yes & 29 & 51.8 \\
\hline \multicolumn{3}{|c|}{ Participation in decision } \\
\hline $\mathrm{No}$ & 38 & 67.9 \\
\hline Yes & 18 & 32.1 \\
\hline \multicolumn{3}{|c|}{ Training facilities } \\
\hline No & 24 & 42.9 \\
\hline Yes & 32 & 57.1 \\
\hline \multicolumn{3}{|c|}{ Autonomy in work } \\
\hline No & 37 & 66.1 \\
\hline Yes & 19 & 33.9 \\
\hline \multicolumn{3}{|c|}{ Gender discrimination } \\
\hline No & 42 & 75.0 \\
\hline Yes & 14 & 25.0 \\
\hline \multicolumn{3}{|c|}{ Enough working hour } \\
\hline No & 27 & 48.2 \\
\hline Yes & 29 & 51.8 \\
\hline \multicolumn{3}{|c|}{ Chance of promotion } \\
\hline No & 31 & 55.4 \\
\hline Yes & 25 & 44.6 \\
\hline \multicolumn{3}{|c|}{ Increase knowledge \& Capacity } \\
\hline No & 3 & 5.4 \\
\hline Yes & 53 & 96.6 \\
\hline \multicolumn{3}{|c|}{ Practice in MBO (Management by Objective) } \\
\hline No & 19 & 33.9 \\
\hline Yes & 37 & 66.1 \\
\hline
\end{tabular}

According to Table 2, employee whose age is 30-35(80.8\%, p<0. 013), works as officer $(93.3 \%$, $\mathrm{p}<0.001)$, draw salary $\leq 2500(91.7 \%$, $\mathrm{p}<.00001)$, family income is $45000-65000(84.2 \%, \mathrm{p}<.004)$ and works in autonomous environment $(88.0 \%, \mathrm{p}<.004)$ cannot participate in decision making. On the contrary employees with age $>35(66.7 \%)$, work as SPO/SEO/Grade-I $(75.0 \%)$, draw salary $>45,000(70.6 \%)$ and work in a participative environment $(48.4 \%)$ and have available opportunity to participate in decision making. 
Table 2: Association between demographic variables and Participation in decision making

\begin{tabular}{|c|c|c|c|}
\hline \multirow[t]{2}{*}{ Variables } & \multicolumn{2}{|c|}{ Participation in Decision Making } & \multirow[t]{2}{*}{ P Values } \\
\hline & No & Yes & \\
\hline \multicolumn{4}{|l|}{ Gender } \\
\hline Male & $35(70.0 \%)$ & $15(30.0 \%)$ & 0.322 \\
\hline Female & $3(50.0 \%)$ & $3(50.0 \%)$ & \\
\hline \multicolumn{4}{|c|}{ Age of the respondents (in years) } \\
\hline$<30$ & $13(72.2 \%)$ & $5(27.8 \%)$ & .013 \\
\hline $30-35$ & $21(80.8 \%)$ & $5(19.2 \%)$ & \\
\hline$>35$ & $4(33.3 \%)$ & $8(66.7 \%)$ & \\
\hline \multicolumn{4}{|c|}{ Religion of the respondents } \\
\hline Islam & $34(66.7 \%)$ & $17(33.3 \%)$ & .542 \\
\hline Hindu & $4(80 \%)$ & $1(20 \%)$ & \\
\hline \multicolumn{4}{|c|}{ Education of the respondents } \\
\hline Honors degree & $4(80 \%)$ & $1(20 \%)$ & .542 \\
\hline Master degree & $34(66.7 \%)$ & $14(33.3 \%)$ & \\
\hline \multicolumn{4}{|c|}{ Designation of the respondents } \\
\hline $\mathrm{TAO} / \mathrm{AO} / \mathrm{JO} / \mathrm{ACO}$ & $19(79.2 \%)$ & $5(20.8)$ & .001 \\
\hline Officer & $14(93.3 \%)$ & $1(6.7 \%)$ & \\
\hline SO/PO/EO/Grade- ii & $3(33.3 \%)$ & $6(66.7 \%)$ & \\
\hline SPO/SEO/Grade-i & $2(25.0 \%)$ & $6(75.0 \%)$ & \\
\hline \multicolumn{4}{|c|}{ Salary of the respondents } \\
\hline$\leq 2500$ & $11(91.7 \%)$ & $1(8.3 \%)$ & .000 \\
\hline $25,001-35,000$ & $15(88.2 \%)$ & $2(11.8 \%)$ & \\
\hline $35,001-45,000$ & $7(70.0 \%)$ & $3(30.3 \%)$ & \\
\hline$>45,000$ & $5(29.4)$ & $12(70.6 \%)$ & \\
\hline \multicolumn{4}{|c|}{ Family member of the respondents } \\
\hline$<<3$ & $11(64.7 \%)$ & $6(35.3 \%)$ & .714 \\
\hline 4 & $10(62.5 \%)$ & $6(37.5)$ & \\
\hline$>4$ & $17(73.9 \%)$ & $6(26.1 \%)$ & \\
\hline \multicolumn{4}{|c|}{ Marital status of the respondents } \\
\hline Married & $30(69.8 \%)$ & $13(30.2 \%)$ & .578 \\
\hline Unmarried & $8(61.5 \%)$ & $5(38.5)$ & \\
\hline \multicolumn{4}{|c|}{ Geographic Location of the respondents } \\
\hline Rural & $4(80 \%)$ & $1(20 \%)$ & .669 \\
\hline Semi- Urban & $25(69.4 \%)$ & $11(30.6 \%)$ & \\
\hline Urban & $9(60 \%)$ & $6(40 \%)$ & \\
\hline \multicolumn{4}{|c|}{ Family income of the respondents } \\
\hline$<45000$ & $14(82.4 \%)$ & $3(17.6 \%)$ & .004 \\
\hline $45000-65000$ & $16(84.2 \%)$ & $3(15.8 \%)$ & \\
\hline$>65000$ & $8(40 \%)$ & $12(60.0 \%)$ & \\
\hline \multicolumn{4}{|c|}{ Service period of the respondents } \\
\hline$<36$ & $17(70.8 \%)$ & $7(29.2 \%)$ & .855 \\
\hline $36-60$ & $11(68.8 \%)$ & $5(31.2 \%)$ & \\
\hline$>60$ & $10(62.5 \%)$ & $6(37.5 \%)$ & \\
\hline \multicolumn{4}{|l|}{ Working Environment } \\
\hline Participative & $16(51.6 \%)$ & $15(48.4 \%)$ & .004 \\
\hline Autonomy & $22(88.0 \%)$ & $3(12.0 \%)$ & \\
\hline
\end{tabular}


In table 3, it is surprisingly found that there is no significant association between demographic characteristics and availability of tools \&resources. But working environment (.113) is close to significance value.

Table 3: Association between demographic variables and Availability of Tools \& Resources

\begin{tabular}{|c|c|c|c|}
\hline \multirow[t]{2}{*}{ Variables } & \multicolumn{2}{|c|}{ Availability of Tools\& Resources } & \multirow[t]{2}{*}{ P Values } \\
\hline & No & Yes & \\
\hline \multicolumn{4}{|l|}{ Gender } \\
\hline Male & $24(48.0 \%)$ & $26(52.0 \%)$ & .926 \\
\hline Female & $3(50.0 \%)$ & $3(50.3 \%)$ & \\
\hline \multicolumn{4}{|c|}{ Age of the respondents (in years) } \\
\hline$<30$ & $9(50.0 \%)$ & $9(50.0 \%)$ & .877 \\
\hline $30-35$ & $13(50.0 \%)$ & $13(50.0 \%)$ & \\
\hline$>35$ & $5(41.7 \%)$ & $7(58.3 \%)$ & \\
\hline \multicolumn{4}{|c|}{ Religion of the respondents } \\
\hline Islam & $25(49.0 \%)$ & $26(51.0 \%)$ & .700 \\
\hline Hindu & $2(40.0 \%)$ & $3(60.0 \%)$ & \\
\hline \multicolumn{4}{|c|}{ Education of the respondents } \\
\hline Honors degree & $3(60.0 \%)$ & $2(40.0 \%)$ & .580 \\
\hline Master degree & $24(47.1 \%)$ & $27(52.9 \%)$ & \\
\hline \multicolumn{4}{|c|}{ Designation of the respondents } \\
\hline $\mathrm{TAO} / \mathrm{AO} / \mathrm{JO} / \mathrm{ACO}$ & $10(41.7 \%)$ & $14(58.3 \%)$ & .223 \\
\hline Officer & $10(66.7 \%)$ & $5(33.3 \%)$ & \\
\hline SO/PO/EO/Grade- ii & $5(55.6 \%)$ & $4(44.4 \%)$ & \\
\hline SPO/SEO/Grade-i & $2(25.0 \%)$ & $6(75.0 \%)$ & \\
\hline \multicolumn{4}{|c|}{ Salary of the respondents } \\
\hline$\leq 2500$ & $6(50.0 \%)$ & $6(50.0 \%)$ & .915 \\
\hline $25,001-35,000$ & $9(52.9 \%)$ & $8(47.1 \%)$ & \\
\hline $35,001-45,000$ & $5(50.0 \%)$ & $5(50.0 \%)$ & \\
\hline$>45,000$ & $7(41.2 \%)$ & $10(58.8 \%)$ & \\
\hline \multicolumn{4}{|c|}{ Family member of the respondents } \\
\hline$<<3$ & $11(64.7 \%)$ & $6(35.3 \%)$ & .254 \\
\hline 4 & $7(43.8 \%)$ & $9(56.2 \%)$ & \\
\hline$>4$ & $9(39.1 \%)$ & $14(60.9 \%)$ & \\
\hline \multicolumn{4}{|c|}{ Marital status of the respondents } \\
\hline Married & $21(48.8 \%)$ & $22(51.2)$ & .856 \\
\hline Unmarried & $6(46.2 \%)$ & $7(53.8 \%)$ & \\
\hline \multicolumn{4}{|c|}{ Geographic Location of the respondents } \\
\hline Rural & $2(40.0 \%)$ & $3(60.0 \%)$ & .326 \\
\hline Semi- Urban & $20(55.6 \%)$ & $16(44.4 \%)$ & \\
\hline Urban & $5(33.3 \%)$ & $10(66.7 \%)$ & \\
\hline \multicolumn{4}{|c|}{ Family income of the respondents } \\
\hline$<45000$ & $9(52.9 \%)$ & $8(47.1 \%)$ & .657 \\
\hline $45000-65000$ & $10(52.6 \%)$ & $9(47.4)$ & \\
\hline$>65000$ & $8(40.0 \%)$ & $12(60.0 \%)$ & \\
\hline \multicolumn{4}{|c|}{ Service period of the respondents } \\
\hline$<36$ & $11(45.8 \%)$ & $13(54.2 \%)$ & .953 \\
\hline $36-60$ & $8(50.0 \%)$ & $8(50.0 \%)$ & \\
\hline$>60$ & $8(50.0 \%)$ & $8(50.0 \%)$ & \\
\hline \multicolumn{4}{|l|}{ Working Environment } \\
\hline Participative & $12(38.7 \%)$ & $19(63.3 \%)$ & .113 \\
\hline Autonomy & $15(60.0 \%)$ & $10(40.0 \%)$ & \\
\hline
\end{tabular}


From the table 4, it has been found that, all top-level employees $(100 \%, \mathrm{p}<0.004)$ get appropriate training facilities. Besides employees, who draw the highest salary $(88.2 \%, \mathrm{p}<0.011)$, have more family income $(80.0 \%, \mathrm{p}<0.035)$ and work in a participative environment $(83.9 \%, \mathrm{p}<0.000)$ are also exceedingly satisfied with the training facility. But employees who work as an officer $(73.3 \%)$ and in the autonomous environment $(76.0 \%)$, get salary $25,001-35,000(64.7 \%)$ and have family income $45000-65000$ (57.9\%) do not get proper training facilities.

Table 4: Association between demographic variables and Training Facilities

\begin{tabular}{|c|c|c|c|}
\hline \multirow[t]{2}{*}{ Variables } & \multicolumn{2}{|c|}{ Training Facilities } & \multirow[t]{2}{*}{ P values } \\
\hline & No & Yes & \\
\hline \multicolumn{4}{|l|}{ Gender } \\
\hline Male & $23(46.0 \%)$ & $27(54.0 \%)$ & .170 \\
\hline Female & $1(16.7 \%)$ & $5(83.3 \%)$ & \\
\hline \multicolumn{4}{|c|}{ Age of the respondents (in years) } \\
\hline$<30$ & $8(44.4 \%)$ & $10(55.6 \%)$ & .346 \\
\hline $30-35$ & $13(50.0 \%)$ & $13(50.0 \%)$ & \\
\hline$>35$ & $3(25.0 \%)$ & $9(75.0 \%)$ & \\
\hline \multicolumn{4}{|c|}{ Religion of the respondents } \\
\hline Islam & $22(43.1 \%)$ & $29(56.9 \%)$ & .892 \\
\hline Hindu & $2(40.0 \%)$ & $3(60.0 \%)$ & \\
\hline \multicolumn{4}{|c|}{ Education of the respondents } \\
\hline Honors degree & $4(80.0 \%)$ & $1(20.0 \%)$ & .079 \\
\hline Master degree & $20(39.1 \%)$ & $31(60.8 \%)$ & \\
\hline \multicolumn{4}{|c|}{ Designation of the respondents } \\
\hline $\mathrm{TAO} / \mathrm{AO} / \mathrm{JO} / \mathrm{ACO}$ & $11(45.8 \%)$ & $13(54.2 \%)$ & .004 \\
\hline Officer & $11(73.3 \%)$ & $4(26.7 \%)$ & \\
\hline $\mathrm{SO} / \mathrm{PO} / \mathrm{EO} /$ Grade- ii & $2(22.2 \%)$ & $7(77.8 \%)$ & \\
\hline SPO/SEO/Grade-i & $0(0.0 \%)$ & $8(100.0 \%)$ & \\
\hline \multicolumn{4}{|c|}{ Salary of the respondents } \\
\hline$\leq 2500$ & $7(58.3 \%)$ & $5(41.7)$ & .011 \\
\hline $25,001-35,000$ & $11(64.7 \%)$ & $6(35.3 \%)$ & \\
\hline $35,001-45,000$ & $4(40.0 \%)$ & $6(60.0 \%)$ & \\
\hline$>45,000$ & $2(11.8 \%)$ & $15(88.2 \%)$ & \\
\hline \multicolumn{4}{|c|}{ Family member of the respondents } \\
\hline$<<3$ & $10(58.8 \%)$ & $7(41.2 \%)$ & .199 \\
\hline 4 & $7(43.8 \%)$ & $9(56.2 \%)$ & \\
\hline$>4$ & $7(30.4 \%)$ & $16(69.6 \%)$ & \\
\hline \multicolumn{4}{|c|}{ Marital status of the respondents } \\
\hline Married & $21(48.8 \%)$ & $22(51.2 \%)$ & .100 \\
\hline Unmarried & $3(23.1 \%)$ & $10(76.9 \%)$ & \\
\hline \multicolumn{4}{|c|}{ Geographic Location of the respondents } \\
\hline Rural & $4(80.0 \%)$ & $1(20.0 \%)$ & .108 \\
\hline Semi- Urban & $16(44.4 \%)$ & $20(55.6 \%)$ & \\
\hline Urban & $4(26.7 \%)$ & $11(73.3 \%)$ & \\
\hline \multicolumn{4}{|c|}{ Family income of the respondents } \\
\hline$<45000$ & $9(52.9 \%)$ & $8(47.1 \%)$ & .035 \\
\hline $45000-65000$ & $11(57.9 \%)$ & $8(42.1 \%)$ & \\
\hline$>65000$ & $4(20.0 \%)$ & $16(80.0 \%)$ & \\
\hline \multicolumn{4}{|c|}{ Service period of the respondents } \\
\hline$<36$ & $8(33.3 \%)$ & $16(66.7 \%)$ & .166 \\
\hline $36-60$ & $6(37.5 \%)$ & $10(62.5 \%)$ & \\
\hline$>60$ & $10(62.5 \%)$ & $6(37.5 \%)$ & \\
\hline \multicolumn{4}{|l|}{ Working Environment } \\
\hline Participative & $5(16.1 \%)$ & $26(83.9 \%)$ & .000 \\
\hline Autonomy & $19(76.0 \%)$ & $6(24.0 \%)$ & \\
\hline
\end{tabular}


According to the table 5, an employee with age $<30(83.3 \%$, p $<0.016)$ who works as an officer $(93.3 \%, \mathrm{p}<0.037)$ cannot enjoy autonomy in his/her working area. On the other hand, an employee with age $>35(66.7 \%)$ who works as an SPO/SEO/Grade-I (62.5\%) are able to practice atomic power.

Table 5: Association between demographic variables and Autonomy in working area

\begin{tabular}{|c|c|c|c|}
\hline \multirow[t]{2}{*}{ Variables } & \multicolumn{2}{|c|}{ Autonomy in Working Area } & \multirow[t]{2}{*}{$P$ values } \\
\hline & No & Yes & \\
\hline \multicolumn{4}{|l|}{ Gender } \\
\hline Male & $35(70.0 \%)$ & $15(30.0 \%)$ & .73 \\
\hline Female & $2(33.3 \%)$ & $4(66.7 \%)$ & \\
\hline \multicolumn{4}{|c|}{ Age of the respondents (in years) } \\
\hline$<30$ & $15(83.3 \%)$ & $3(16.7 \%)$ & .016 \\
\hline $30-35$ & $18(69.2 \%)$ & $8(30.8 \%)$ & \\
\hline$>35$ & $4(33.3 \%)$ & $8(66.7 \%)$ & \\
\hline \multicolumn{4}{|c|}{ Religion of the respondents } \\
\hline Islam & $33(64.7 \%)$ & $18(35.3 \%)$ & .491 \\
\hline Hindu & $4(80.0 \%)$ & $1(20.0 \%)$ & \\
\hline \multicolumn{4}{|c|}{ Education of the respondents } \\
\hline Honors degree & $3(60.0 \%)$ & $2(40.0 \%)$ & .764 \\
\hline Master degree & $34(66.7 \%)$ & $17(33.3 \%)$ & \\
\hline \multicolumn{4}{|c|}{ Designation of the respondents } \\
\hline $\mathrm{TAO} / \mathrm{AO} / \mathrm{JO} / \mathrm{ACO}$ & $15(62.5 \%)$ & $9(37.5 \%)$ & .037 \\
\hline Officer & $14(93.3 \%)$ & $1(6.7 \%)$ & \\
\hline SO/PO/EO/Grade- ii & $5(55.6 \%)$ & $4(44.4 \%)$ & \\
\hline SPO/SEO/Grade-i & $3(37.5 \%)$ & $5(62.5 \%)$ & \\
\hline \multicolumn{4}{|c|}{ Salary of the respondents } \\
\hline$\leq 2500$ & $10(83.3 \%)$ & $2(16.7 \%)$ & .348 \\
\hline $25,001-35,000$ & $12(70.6 \%)$ & $5(29.4 \%)$ & \\
\hline $35,001-45,000$ & $5(50.0 \%)$ & $5(50.0 \%)$ & \\
\hline$>45,000$ & $10(58.8 \%)$ & $7(41.2 \%)$ & \\
\hline \multicolumn{4}{|c|}{ Family member of the respondents } \\
\hline$<<3$ & $12(70.6 \%)$ & $5(29.4 \%)$ & 269 \\
\hline 4 & $8(50.0 \%)$ & $8(50.0 \%)$ & \\
\hline$>4$ & $17(73.9 \%)$ & $6(26.1 \%)$ & \\
\hline \multicolumn{4}{|c|}{ Marital status of the respondents } \\
\hline Married & $27(62.8 \%)$ & $16(37.2 \%)$ & .346 \\
\hline Unmarried & $10(76.9 \%)$ & $3(23.1 \%)$ & \\
\hline \multicolumn{4}{|c|}{ Geographic Location of the respondents } \\
\hline Rural & $4(80.0 \%)$ & $1(20.0 \%)$ & .169 \\
\hline Semi- Urban & $26(72.2 \%)$ & $10(27.8 \%)$ & \\
\hline Urban & $7(46.7 \%)$ & $8(53.3 \%)$ & \\
\hline \multicolumn{4}{|c|}{ Family income of the respondents } \\
\hline$<45000$ & $12(70.6 \%)$ & $5(29.4 \%)$ & .888 \\
\hline $45000-65000$ & $12(63.2 \%)$ & $7(36.8 \%)$ & \\
\hline$>65000$ & $13(65.0 \%)$ & $7(35.0 \%)$ & \\
\hline \multicolumn{4}{|c|}{ Service period of the respondents } \\
\hline$<36$ & $19(79.2 \%)$ & $5(20.8 \%)$ & .152 \\
\hline $36-60$ & $10(62.5 \%)$ & $6(37.5 \%)$ & \\
\hline$>60$ & $8(50.0 \%)$ & $8(50.0 \%)$ & \\
\hline \multicolumn{4}{|c|}{ Working Environment } \\
\hline Participative & $19(61.3 \%)$ & $12(38.7 \%)$ & .400 \\
\hline Autonomy & $18(72.0 \%)$ & $7(28.0 \%)$ & \\
\hline
\end{tabular}


Table 6 represents that employees who are unmarried $(53.8 \%, \mathrm{p}<0.006)$, service period $<36$ months $(41.7 \%, \mathrm{p}<0.032)$ and work in participative environment $(35.5 \%, \mathrm{p}<0.044)$ face gender discrimination. But employees who are married (83.7\%), service period $>60$ months $(93.8 \%)$ and work in an autonomous environment $(88.0 \%)$ have a positive response regarding gender discrimination.

Table 6: Association between demographic variables and Gender discrimination

\begin{tabular}{|c|c|c|c|}
\hline \multirow[t]{2}{*}{ Variables } & \multicolumn{2}{|c|}{ Gender Discrimination } & \multirow[t]{2}{*}{ P Values } \\
\hline & No & Yes & \\
\hline \multicolumn{4}{|l|}{ Gender } \\
\hline Male & $36(72.0 \%)$ & $14(28.0 \%)$ & .134 \\
\hline Female & $6(100.0 \%)$ & $0(00.0 \%)$ & \\
\hline \multicolumn{4}{|c|}{ Age of the respondents (in years) } \\
\hline$<30$ & $12(66.7 \%)$ & $6(33.3 \%)$ & .302 \\
\hline $30-35$ & $22(84.6 \%)$ & $4(15.4 \%)$ & \\
\hline$>35$ & $8(66.7 \%)$ & $4(33.3 \%)$ & \\
\hline \multicolumn{4}{|c|}{ Religion of the respondents } \\
\hline Islam & $38(47.5 \%)$ & $13(25.5 \%)$ & .787 \\
\hline Hindu & $4(80.0 \%)$ & $1(20.0 \%)$ & \\
\hline \multicolumn{4}{|c|}{ Education of the respondents } \\
\hline Honors degree & $4(80.0 \%)$ & $1(20.0 \%)$ & .787 \\
\hline Master degree & $38(47.5 \%)$ & $13(25.5 \%)$ & \\
\hline \multicolumn{4}{|c|}{ Designation of the respondents } \\
\hline $\mathrm{TAO} / \mathrm{AO} / \mathrm{JO} / \mathrm{ACO}$ & $19(79.2 \%)$ & $5(20.8 \%)$ & .846 \\
\hline Officer & $10(66.7 \%)$ & $5(33.3 \%)$ & \\
\hline $\mathrm{SO} / \mathrm{PO} / \mathrm{EO} /$ Grade- $\mathrm{ii}$ & $7(77.8 \%)$ & $2(22.2 \%)$ & \\
\hline SPO/SEO/Grade-i & $6(75.0 \%)$ & $2(25.0 \%)$ & \\
\hline \multicolumn{4}{|c|}{ Salary of the respondents } \\
\hline$\leq 2500$ & $10(83.3 \%)$ & $2(16.7 \%)$ & .510 \\
\hline $25,001-35,000$ & $14(82.4 \%)$ & $3(17.6 \%)$ & \\
\hline $35,001-45,000$ & $6(60.0 \%)$ & $4(40.0 \%)$ & \\
\hline$>45,000$ & $12(70.6 \%)$ & $5(29.4 \%)$ & \\
\hline \multicolumn{4}{|c|}{ Family member of the respondents } \\
\hline$<<3$ & $16(94.1 \%)$ & $1(5.9 \%)$ & .082 \\
\hline 4 & $10(62.5 \%)$ & $6(37.5 \%)$ & \\
\hline$>4$ & $16(69.6 \%)$ & $7(30.4 \%)$ & \\
\hline \multicolumn{4}{|c|}{ Marital status of the respondents } \\
\hline Married & $36(83.7 \%)$ & $7(16.3 \%)$ & .006 \\
\hline Unmarried & $6(46.2 \%)$ & $7(53.8 \%)$ & \\
\hline \multicolumn{4}{|c|}{ Geographic Location of the respondents } \\
\hline Rural & $4(80.0 \%)$ & $1(20.0 \%)$ & .417 \\
\hline Semi- Urban & $25(69.4 \%)$ & $11(30.6 \%)$ & \\
\hline Urban & $13(86.7 \%)$ & $2(13.3 \%)$ & \\
\hline \multicolumn{4}{|c|}{ Family income of the respondents } \\
\hline$<45000$ & $15(88.2 \%)$ & $2(11.8 \%)$ & .318 \\
\hline $45000-65000$ & $13(68.4 \%)$ & $6(31.6 \%)$ & \\
\hline$>65000$ & $14(70.0 \%)$ & $6(30.0 \%)$ & \\
\hline \multicolumn{4}{|c|}{ Service period of the respondents } \\
\hline$<36$ & $14(58.3 \%)$ & $10(41.7 \%)$ & .032 \\
\hline $36-60$ & $13(81.2 \%)$ & $3(18.8 \%)$ & \\
\hline$>60$ & $15(93.8 \%)$ & $1(6.2 \%)$ & \\
\hline \multicolumn{4}{|l|}{ Working Environment } \\
\hline Participative & $20(64.5 \%)$ & $11(35.5 \%)$ & .044 \\
\hline Autonomy & $22(88.0 \%)$ & $3(12.0 \%)$ & \\
\hline
\end{tabular}


Table 7 illustrates that all top-level employees $(100 \%, \mathrm{p}<0.003)$ and employees whose family income $>65000(80.0 \%, \mathrm{p}<0.007)$ and work in a participative environment $(74.2 \%, \mathrm{p}<0.007)$ get enough working hour. But employees work as an officer (80.0\%), family income $<45000(64.7 \%)$ and work in an autonomous environment (76.0\%) do not get enough working hour.

Table 7: Association between demographic variables and Working hour

\begin{tabular}{|c|c|c|c|}
\hline \multirow[t]{2}{*}{ Variables } & \multicolumn{2}{|c|}{ Working Hour } & \multirow[t]{2}{*}{ P Values } \\
\hline & No & Yes & \\
\hline \multicolumn{4}{|l|}{ Gender } \\
\hline Male & $25(50.0 \%)$ & $25(50.0 \%)$ & .440 \\
\hline Female & $2(33.3 \%)$ & $4(66.7 \%)$ & \\
\hline \multicolumn{4}{|c|}{ Age of the respondents (in years) } \\
\hline$<30$ & $9(50.0 \%)$ & $9(50.0 \%)$ & .492 \\
\hline $30-35$ & $14(53.8 \%)$ & $12(46.2 \%)$ & \\
\hline$>35$ & $4(33.3 \%)$ & $8(66.7 \%)$ & \\
\hline \multicolumn{4}{|c|}{ Religion of the respondents } \\
\hline Islam & $25(49.0 \%)$ & $26(51.0 \%)$ & .700 \\
\hline Hindu & $2(40.0 \%)$ & $3(60.0 \%)$ & \\
\hline \multicolumn{4}{|c|}{ Education of the respondents } \\
\hline Honors degree & $3(60.0 \%)$ & $2(40.0 \%)$ & .580 \\
\hline Master degree & $24(47.1 \%)$ & $27(52.9 \%)$ & \\
\hline \multicolumn{4}{|c|}{ Designation of the respondents } \\
\hline $\mathrm{TAO} / \mathrm{AO} / \mathrm{JO} / \mathrm{ACO}$ & $10(41.7 \%)$ & $14(58.3 \%)$ & .003 \\
\hline Officer & $12(80.0 \%)$ & $3(20.0 \%)$ & \\
\hline $\mathrm{SO} / \mathrm{PO} / \mathrm{EO} /$ Grade- ii & $5(55.6 \%)$ & $4(44.4 \%)$ & \\
\hline SPO/SEO/Grade-i & $0(00.0 \%)$ & $8(100.0 \%)$ & \\
\hline \multicolumn{4}{|c|}{ Salary of the respondents } \\
\hline$\leq 2500$ & $7(58.3 \%)$ & $5(41.7 \%)$ & .082 \\
\hline $25,001-35,000$ & $9(52.9 \%)$ & $8(47.1 \%)$ & \\
\hline $35,001-45,000$ & $7(70.0 \%)$ & $3(30.0 \%)$ & \\
\hline$>45,000$ & $4(23.5 \%)$ & $13(76.5 \%)$ & \\
\hline \multicolumn{4}{|c|}{ Family member of the respondents } \\
\hline$<<3$ & $11(64.7 \%)$ & $6(35.3 \%)$ & .075 \\
\hline 4 & $9(56.2 \%)$ & $7(43.8 \%)$ & \\
\hline$>4$ & $7(30.4 \%)$ & $16(69.6 \%)$ & \\
\hline \multicolumn{4}{|c|}{ Marital status of the respondents } \\
\hline Married & $20(46.5 \%)$ & $23(53.5 \%)$ & .642 \\
\hline Unmarried & $7(53.8 \%)$ & $6(46.2 \%)$ & \\
\hline \multicolumn{4}{|c|}{ Geographic Location of the respondents } \\
\hline Rural & $3(60.0 \%)$ & $2(40.0 \%)$ & .385 \\
\hline Semi- Urban & $19(52.8 \%)$ & $17(47.2 \%)$ & \\
\hline Urban & $5(33.3 \%)$ & $10(66.7 \%)$ & \\
\hline \multicolumn{4}{|c|}{ Family income of the respondents } \\
\hline$<45000$ & $11(64.7 \%)$ & $6(35.3 \%)$ & .007 \\
\hline $45000-65000$ & $12(63.2 \%)$ & $7(36.8 \%)$ & \\
\hline$>65000$ & $4(20.0 \%)$ & $16(80.0 \%)$ & \\
\hline \multicolumn{4}{|c|}{ Service period of the respondents } \\
\hline$<36$ & $10(41.7 \%)$ & $14(58.3 \%)$ & .397 \\
\hline $36-60$ & $7(43.8 \%)$ & $9(56.2 \%)$ & \\
\hline$>60$ & $10(62.5 \%)$ & $6(37.5 \%)$ & \\
\hline \multicolumn{4}{|l|}{ Working Environment } \\
\hline Participative & $8(25.8 \%)$ & $23(74.2 \%)$ & .000 \\
\hline Autonomy & $19(76.0 \%)$ & $6(24.0 \%)$ & \\
\hline
\end{tabular}


From table 8 it has been found that, employees age between 30 and $35(80.8 \%, \mathrm{p}<0.002)$, work as an officer $(86.7 \%, \mathrm{p}<0.006)$ and in the autonomous environment $(72.0 \%, \mathrm{p}<0.024)$ do not get a right promotion. On the other hand, employees of both $<30$ and $>35$ age $(66.7 \%)$, work as SPO/SEO/Grade-I (87.5\%) and in participative environment $(58.1 \%)$ report that they have a proper promotion.

Table 8: Association between demographic variables and Chance of promotion

\begin{tabular}{|c|c|c|c|}
\hline \multirow[t]{2}{*}{ Variables } & \multicolumn{2}{|c|}{ Chance of Promotion } & \multirow[t]{2}{*}{ P Values } \\
\hline & No & Yes & \\
\hline \multicolumn{4}{|l|}{ Gender } \\
\hline Male & $29(58.0 \%)$ & $21(42.0 \%)$ & .251 \\
\hline Female & $2(33.3 \%)$ & $4(66.7 \%)$ & \\
\hline \multicolumn{4}{|c|}{ Age of the respondents (in years) } \\
\hline$<30$ & $6(33.3 \%)$ & $12(66.7 \%)$ & .002 \\
\hline $30-35$ & $21(80.8 \%)$ & $5(19.2 \%)$ & \\
\hline$>35$ & $4(33.3 \%)$ & $8(66.7 \%)$ & \\
\hline \multicolumn{4}{|c|}{ Religion of the respondents } \\
\hline Islam & $29(56.9 \%)$ & $22(43.1 \%)$ & .469 \\
\hline Hindu & $2(40.0 \%)$ & $3(60.0 \%)$ & \\
\hline \multicolumn{4}{|c|}{ Education of the respondents } \\
\hline Honors degree & $4(80.0 \%)$ & $1(20.0 \%)$ & .245 \\
\hline Master degree & $27(52.9 \%)$ & $24(47.1 \%)$ & \\
\hline \multicolumn{4}{|c|}{ Designation of the respondents } \\
\hline $\mathrm{TAO} / \mathrm{AO} / \mathrm{JO} / \mathrm{ACO}$ & $13(54.2 \%)$ & $11(45.8 \%)$ & .006 \\
\hline Officer & $13(86.7 \%)$ & $2(13.3 \%)$ & \\
\hline $\mathrm{SO} / \mathrm{PO} / \mathrm{EO} /$ Grade- ii & $4(44.4 \%)$ & $5(55.6 \%)$ & \\
\hline SPO/SEO/Grade-i & $1(12.5 \%)$ & $7(87.5 \%)$ & \\
\hline \multicolumn{4}{|c|}{ Salary of the respondents } \\
\hline$\leq 2500$ & $5(41.7 \%)$ & $7(58.3 \%)$ & .10 \\
\hline $25,001-35,000$ & $14(82.4 \%)$ & $3(17.6 \%)$ & \\
\hline $35,001-45,000$ & $7(70.0 \%)$ & $3(30.0 \%)$ & \\
\hline$>45,000$ & $5(29.4 \%)$ & $12(70.6 \%)$ & \\
\hline \multicolumn{4}{|c|}{ Family member of the respondents } \\
\hline$<<3$ & $9(52.9 \%)$ & $8(47.1 \%)$ & .793 \\
\hline 4 & $10(62.5 \%)$ & $6(67.5 \%)$ & \\
\hline$>4$ & $12(52.2 \%)$ & $11(47.8 \%)$ & \\
\hline \multicolumn{4}{|c|}{ Marital status of the respondents } \\
\hline Married & $24(55.8 \%)$ & $19(44.2 \%)$ & .900 \\
\hline Unmarried & $7(53.8 \%)$ & $6(46.2 \%)$ & \\
\hline \multicolumn{4}{|c|}{ Geographic Location of the respondents } \\
\hline Rural & $3(60.0 \%)$ & $2(40.0 \%)$ & .376 \\
\hline Semi- Urban & $22(61.1 \%)$ & $14(38.9 \%)$ & \\
\hline Urban & $6(40.0 \%)$ & $9(60.0 \%)$ & \\
\hline \multicolumn{4}{|c|}{ Family income of the respondents } \\
\hline$<45000$ & $12(70.6 \%)$ & $5(29.4 \%)$ & .169 \\
\hline $45000-65000$ & $11(57.9 \%)$ & $8(42.1 \%)$ & \\
\hline$>65000$ & $8(40.0 \%)$ & $12(60.0 \%)$ & \\
\hline \multicolumn{4}{|c|}{ Service period of the respondents } \\
\hline$<36$ & $11(45.8 \%)$ & $13(54.2 \%)$ & .463 \\
\hline $36-60$ & $10(62.5 \%)$ & $6(37.5 \%)$ & \\
\hline$>60$ & $10(62.5 \%)$ & $6(37.5 \%)$ & \\
\hline \multicolumn{4}{|l|}{ Working Environment } \\
\hline Participative & $13(41.9 \%)$ & $18(58.1 \%)$ & .024 \\
\hline Autonomy & $18(72.0 \%)$ & $7(28.0 \%)$ & \\
\hline
\end{tabular}


Table 9 demonstrates that, all employees who are married $(100.0 \%, \mathrm{p}<0.001)$ and family member 4 and $>4(100.0 \%, \mathrm{p}<0.026)$, almost all-male employees $(98.0 \%, \mathrm{p}<0.001)$ say organizational environment increases their capacity and knowledge.

Table 9: Association between demographic variables and Increasing capacity and Knowledge

\begin{tabular}{|c|c|c|c|}
\hline \multirow[t]{2}{*}{ Variables } & \multicolumn{2}{|c|}{ Increasing Capacity and Knowledge } & \multirow[t]{2}{*}{ P Values } \\
\hline & No & Yes & \\
\hline \multicolumn{4}{|l|}{ Gender } \\
\hline Male & $1(2.0 \%)$ & $49(98.0 \%)$ & .001 \\
\hline Female & $2(33.3 \%)$ & $4(66.7 \%)$ & \\
\hline \multicolumn{4}{|c|}{ Age of the respondents (in years) } \\
\hline$<30$ & $2(11.1 \%)$ & $16(88.9)$ & .373 \\
\hline $30-35$ & $1(3.8 \%)$ & $25(96.2 \%)$ & \\
\hline$>35$ & $0(0 \%)$ & $12(100.0 \%)$ & \\
\hline \multicolumn{4}{|c|}{ Religion of the respondents } \\
\hline Islam & $3(5.9 \%)$ & $48(94.1 \%)$ & .577 \\
\hline Hindu & $0(0 \%)$ & $5(100.0 \%)$ & \\
\hline \multicolumn{4}{|c|}{ Education of the respondents } \\
\hline Honors degree & $0(0 \%)$ & $5(100.0 \%)$ & .577 \\
\hline Master degree & $3(5.9 \%)$ & $48(94.1 \%)$ & \\
\hline \multicolumn{4}{|c|}{ Designation of the respondents } \\
\hline $\mathrm{TAO} / \mathrm{AO} / \mathrm{JO} / \mathrm{ACO}$ & $3(12.5 \%)$ & $21(87.5 \%)$ & .238 \\
\hline Officer & $0(0 \%)$ & $15(100.0 \%)$ & \\
\hline SO/PO/EO/Grade- ii & $0(0 \%)$ & $9(100.0 \%)$ & \\
\hline SPO/SEO/Grade-i & $0(0 \%)$ & $8(100.0 \%)$ & \\
\hline \multicolumn{4}{|c|}{ Salary of the respondents } \\
\hline$\leq 2500$ & $1(8.3 \%)$ & $11(91.7 \%)$ & .658 \\
\hline $25,001-35,000$ & $1(5.9 \%)$ & $16(94.1 \%)$ & \\
\hline $35,001-45,000$ & $1(10.0 \%)$ & $9(90.0 \%)$ & \\
\hline$>45,000$ & $0(0 \%)$ & $17(100.0 \%)$ & \\
\hline \multicolumn{4}{|c|}{ Family member of the respondents } \\
\hline$<<3$ & $3(17.6 \%)$ & $14(82.4 \%)$ & .026 \\
\hline 4 & $0(0 \%)$ & $16(100.0 \%)$ & \\
\hline$>4$ & $0(0 \%)$ & $23(100.0 \%)$ & \\
\hline \multicolumn{4}{|c|}{ Marital status of the respondents } \\
\hline Married & $0(0 \%)$ & $43(100.0 \%)$ & .001 \\
\hline Unmarried & $3(23.1 \%)$ & $10(76.9 \%)$ & \\
\hline \multicolumn{4}{|c|}{ Geographic Location of the respondents } \\
\hline Rural & $0(0 \%)$ & $5(100.0 \%)$ & .267 \\
\hline Semi- Urban & $1(2.8 \%)$ & $35(97.2 \%)$ & \\
\hline Urban & $2(13.3 \%)$ & $13(86.7 \%)$ & \\
\hline \multicolumn{4}{|c|}{ Family income of the respondents } \\
\hline$<45000$ & $1(5.9 \%)$ & $16(94.1 \%)$ & .343 \\
\hline $45000-65000$ & $2(10.5 \%)$ & $17(89.5 \%)$ & \\
\hline$>65000$ & $0(0 \%)$ & $20(100.0 \%)$ & \\
\hline \multicolumn{4}{|c|}{ Service period of the respondents } \\
\hline$<36$ & $2(8.3 \%)$ & $22(91.7 \%)$ & .509 \\
\hline $36-60$ & $1(6.2 \%)$ & $15(93.8 \%)$ & \\
\hline$>60$ & $0(0 \%)$ & $16(100.0 \%)$ & \\
\hline \multicolumn{4}{|l|}{ Working Environment } \\
\hline Participative & $2(6.5 \%)$ & $29(93.5 \%)$ & .685 \\
\hline Autonomy & $1(4.0 \%)$ & $24(96.0 \%)$ & \\
\hline
\end{tabular}


According to table 10, almost all female employees $(83.3 \%, \mathrm{p}<0.007)$ and employees who are unmarried $(61.5 \%, \mathrm{p}<0.016)$, service period $<36$ months $(54.2 \%, \mathrm{p}<0.022)$ and work in participative environment $(51.6 \%, \mathrm{p}<0.002)$ cannot get opportunities to participate in goal setting. On the contrary, employees who are male $(72.0 \%)$, married $(74.4 \%)$, service period3660 and $>60$ months $(81.2 \%)$ and work in autonomous environment $(88.0 \%)$ report that they have an opportunity to participate in goal setting.

Table 10: Association between demographic variables and Practice of MBO

\begin{tabular}{|c|c|c|c|}
\hline \multirow[t]{2}{*}{ Variables } & \multicolumn{2}{|c|}{ Practice of MBO } & \multirow[t]{2}{*}{ P Values } \\
\hline & No & Yes & \\
\hline \multicolumn{4}{|l|}{ Gender } \\
\hline Male & $14(28.0 \%)$ & $36(72.0 \%$ & .007 \\
\hline Female & $5(83.3 \%)$ & $1(16.7 \%)$ & \\
\hline \multicolumn{4}{|c|}{ Age of the respondents (in years) } \\
\hline$<30$ & $7(38.9 \%)$ & $11(61.1 \%)$ & .730 \\
\hline $30-35$ & $9(34.6 \%)$ & $17(65.4 \%)$ & \\
\hline$>35$ & $3(25.0 \%)$ & $9(75.0 \%)$ & \\
\hline \multicolumn{4}{|c|}{ Religion of the respondents } \\
\hline Islam & $16(31.4 \%)$ & $35(68.6 \%)$ & .197 \\
\hline Hindu & $3(60.0 \%)$ & $2(40.0 \%)$ & \\
\hline \multicolumn{4}{|c|}{ Education of the respondents } \\
\hline Honors degree & $0(0 \%)$ & $5(100.0 \%)$ & .093 \\
\hline Master degree & $19(37.3 \%)$ & $32(62.7 \%)$ & \\
\hline \multicolumn{4}{|c|}{ Designation of the respondents } \\
\hline $\mathrm{TAO} / \mathrm{AO} / \mathrm{JO} / \mathrm{ACO}$ & $9(37.5 \%)$ & $15(62.5 \%)$ & .935 \\
\hline Officer & $5(33.3 \%)$ & $10(66.7 \%)$ & \\
\hline SO/PO/EO/Grade- ii & $3(33.3 \%)$ & $6(66.7 \%)$ & \\
\hline SPO/SEO/Grade-i & $2(25.0 \%)$ & $6(75.0 \%)$ & \\
\hline \multicolumn{4}{|c|}{ Salary of the respondents } \\
\hline$\leq 2500$ & $3(25.0 \%)$ & $9(75.0 \%)$ & .232 \\
\hline $25,001-35,000$ & $4(23.5 \%)$ & $13(76.5 \%)$ & \\
\hline $35,001-45,000$ & $6(60.0 \%)$ & $4(40.0 \%)$ & \\
\hline$>45,000$ & $6(35.3 \%)$ & $11(64.7 \%)$ & \\
\hline \multicolumn{4}{|c|}{ Family member of the respondents } \\
\hline$<<3$ & $3(17.6 \%)$ & $14(82.4 \%)$ & .219 \\
\hline 4 & $6(37.5 \%)$ & $10(62.5 \%)$ & \\
\hline$>4$ & $10(43.5 \%)$ & $13(56.5 \%)$ & \\
\hline \multicolumn{4}{|c|}{ Marital status of the respondents } \\
\hline Married & $11(25.6 \%)$ & $32(74.4 \%)$ & .016 \\
\hline Unmarried & $8(61.5 \%)$ & $5(38.5 \%)$ & \\
\hline \multicolumn{4}{|c|}{ Geographic Location of the respondents } \\
\hline Rural & $1(20.0 \%)$ & $4(80.0 \%)$ & .774 \\
\hline Semi- Urban & $13(36.1 \%)$ & $23(63.9 \%)$ & \\
\hline Urban & $5(33.3 \%)$ & $10(66.7 \%)$ & \\
\hline \multicolumn{4}{|c|}{ Family income of the respondents } \\
\hline$<45000$ & $4(23.5 \%)$ & $13(76.5 \%)$ & .497 \\
\hline $45000-65000$ & $8(42.1 \%)$ & $11(57.9 \%)$ & \\
\hline$>65000$ & $7(35.0 \%)$ & $13(65.0 \%)$ & \\
\hline \multicolumn{4}{|c|}{ Service period of the respondents } \\
\hline$<36$ & $13(54.2 \%)$ & $11(45.8 \%)$ & .022 \\
\hline $36-60$ & $3(18.8 \%)$ & $13(81.2 \%)$ & \\
\hline$>60$ & $3(18.8 \%)$ & $13(81.2 \%)$ & \\
\hline
\end{tabular}




\begin{tabular}{|l|c|c|c|}
\hline Working Environment & & & \\
\hline Participative & $16(51.6 \%)$ & $15(48.4 \%)$ & .002 \\
\hline Autonomy & $3(12.0 \%)$ & $22(88.0 \%)$ & \\
\hline
\end{tabular}

Notes:

TAO/AS/JO/AOC= Training Assistant Officer/Assistant Officer/Junior Officer/Assistant Officer Cash SO/PO/EO= Senior Officer/Principal Officer/Executive Officer/Grade- ii

SPO/SEO=Senior Principal Officer/Senior Executive Officer/Grade- $i$

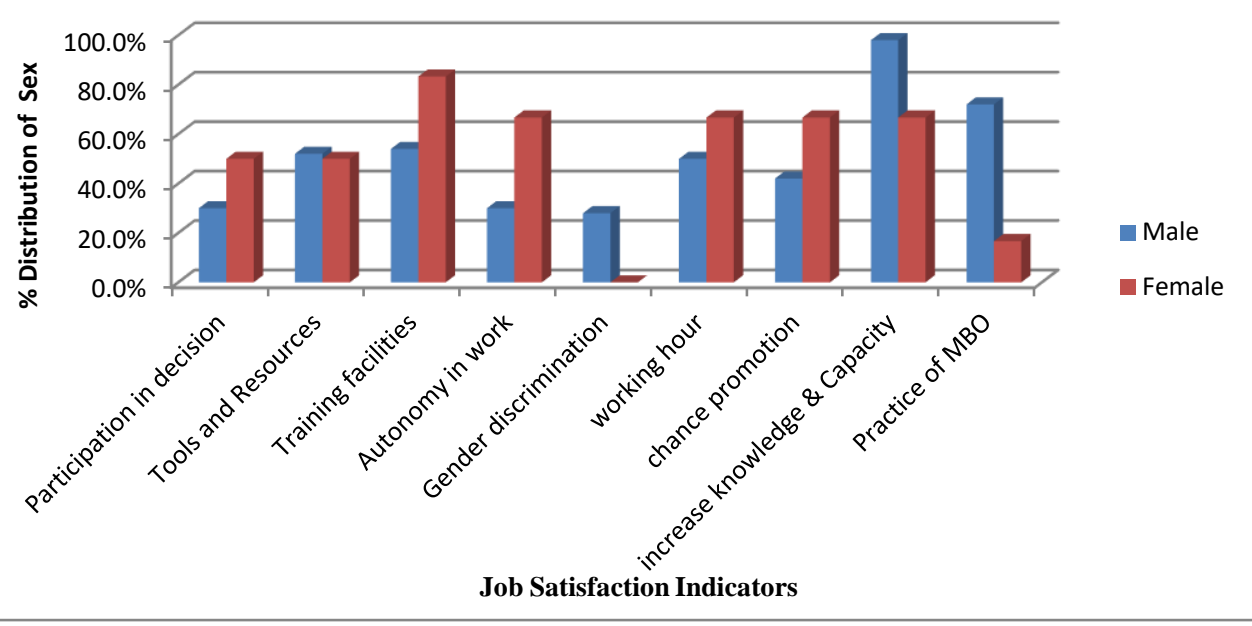

Figure 1: Comparison of percentage distribution of job satisfaction between male and female

According to figure 1, a female is more satisfied than male for the indicators namely participation in decision making, training facilities, work autonomy, working an hour and promotion facilities where the male is more satisfied with tools and resources, gender discrimination, knowledge and capacity and practice of $\mathrm{MBO}$.

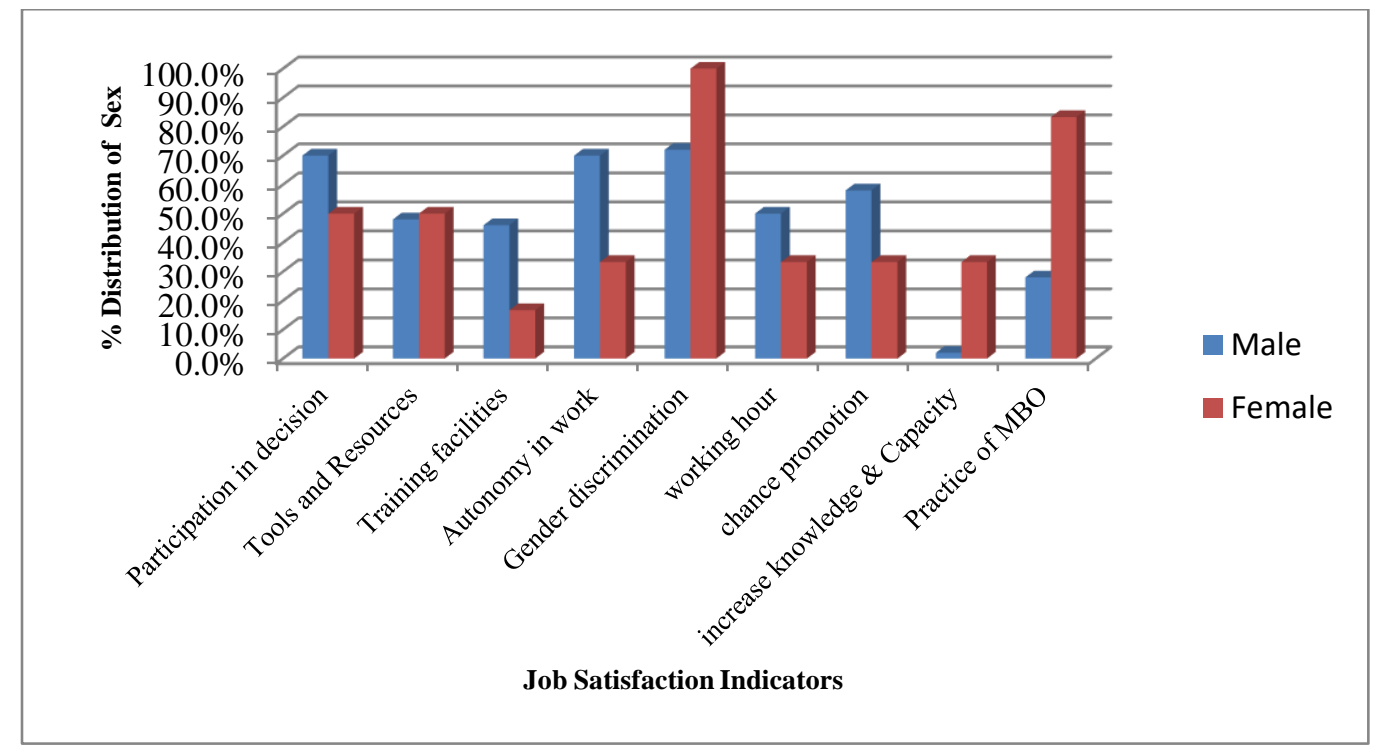

Figure 2: Comparison of percentage distribution of job dissatisfaction between male and female 
From figure 2, it is clear that male is more dissatisfied for participation in decision making, training facilities, work autonomy, working hour and promotion facilities indicators and female is more dissatisfied for tools and resources, gender discrimination, knowledge and capacity and practice of $\mathrm{MBO}$

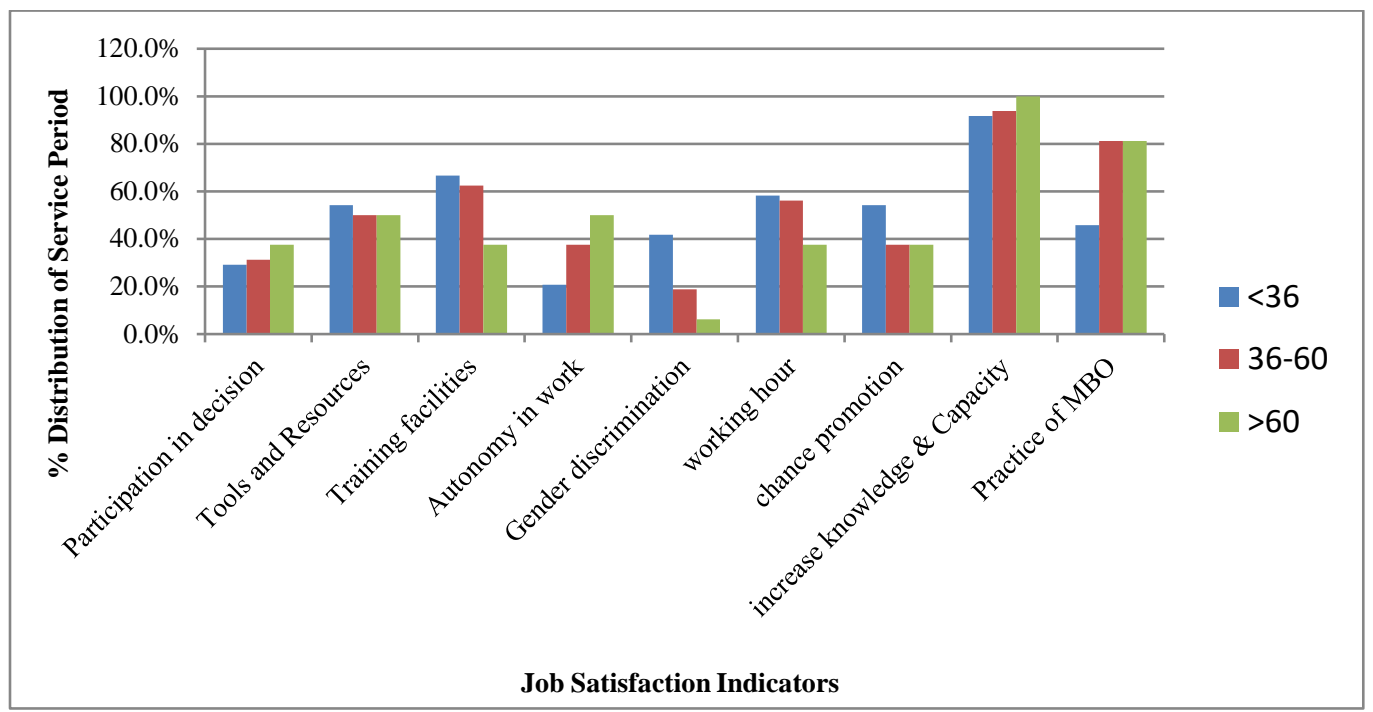

Figure 3: Comparison of percentage distribution of job satisfaction among service period (in month)

Figure 3, shows that new employees are comparatively more satisfied than the seniors and they are more pleased with tools and resources, training, gender discrimination and promotion. The employees with 36-60 months and $>60$ months service period is more pleased for training, gender discrimination, working hour and participation in decision making, job autonomy, increase knowledge and capacity respectively.

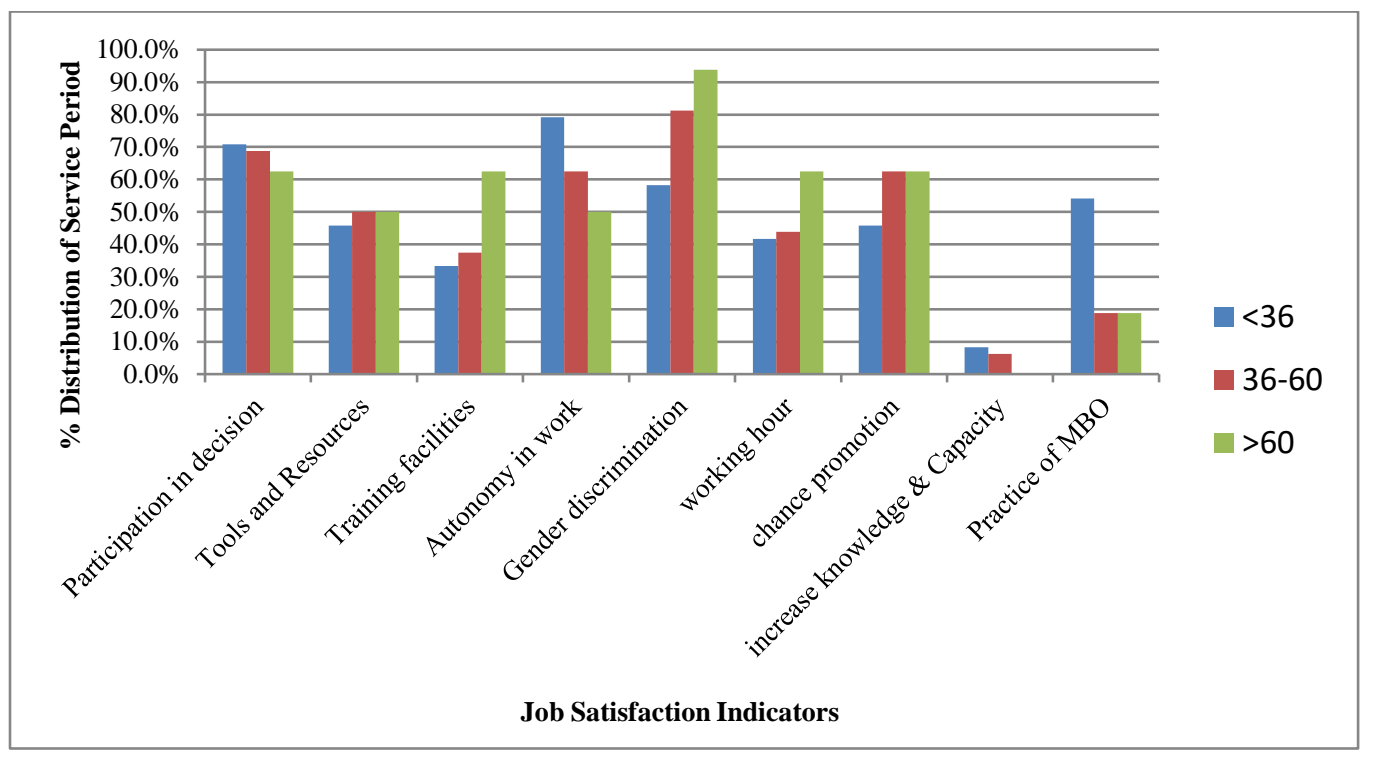

Figure 4: Comparison of percentage distribution of job dissatisfaction among service period (in month) 
Figure 4, illustrates that employee with $<36$ service period is highly dissatisfied for the indicators like as, participation in decision making, autonomy in work, facilities of increasing knowledge and capacity and MBO.

\section{DIscussion}

This study used demographic factors like sex, age, religion, education, designation, salary, family member, marital status, geographic location, family income, service period and working environment to show the association with employees work satisfaction indicators which were take part in decision making, availability of tools and resources, training facilities, autonomy in work, gender discrimination, working hour, chance of promotion, increase knowledge \& capacity, and practice of $\mathrm{MBO}$. According to this study, a significant inter-class relationship was observed between demographic characteristics (sex, age, designation, salary, and family member, marital status, working environment, service period and family income) and satisfaction factors (participation in decision making, training facilities, autonomy in work, gender discrimination, working hour, chance of promotion, increase knowledge \& capacity, practice of $\mathrm{MBO}$ ) and surprisingly availability of tools and resources had no significant relation with any demographic factors. Our findings was partially consistent with the findings of a study by Heidarian et al. (2015) in Iran which found significance relation between demographic characteristics (age, marital status, gender, hire status and years of service) and motivational factors (advancement, recognition, responsibility, education and development, interpersonal relations, equity, pay, job security, recognition, attractiveness of job supervision, organizational policies, working conditions). However, we used Chi-square test in our study where as Heidarian et al. (2015) used correlation analysis. Popoola (2009) revealed job satisfaction and socio-economic factors (i.e. gender, age, marital status, education, job tenure and salary) were significantly correlated with organizational commitment.

This study showed that sex had significance relation with increasing capacity and knowledge, practice of $\mathrm{MBO}$ where different studies revealed different results. Job satisfaction had no relationship with sex and job designation (James, 2020). Talukder et al. (2014) cited that med men employees are much more dissatisfied than their counterpart women, while Rahman et al. (2017) found male is more satisfied than female. Heidarian et al. (2015) found female employees had little opportunity to participate in goal setting and decision making. On the other hand, Budría \& Baleix (2020); Azad et al., (2011); and Ali and Akhter (2009) found that there is no significant difference between male and female employees' job satisfaction.

We found age's significant relation with participation in decision making, autonomy in work, chance promotion while Heidarian et al. (2015) found that job satisfaction indicators like as attractiveness of job, working condition, supervision and organization policies were significantly associated with age. Vickovic \& Morrow (2020) revealed that age had an effect on performance, job satisfaction and turnover. Age, job title and marital status had positive influence on employees work satisfaction (Buker and Dolu, 2010). Rahman et al. (2017) showed that employees of 30-35 age groups had low levels of job satisfaction (76.04\%). Molla, (2015) claimed aged worker had less job satisfaction.

According to this study marital status had significant relation with Gender Discrimination, Increasing Capacity and Knowledge, Practice of MBO. Vickovic \& Morrow (2020) showed that marital status had significant relation with performance, job satisfaction and turnover, Heidarian et al. (2015) found marital status had significant relation with advancement and 
responsibility. Significant differences in job satisfaction was found between single and married (Park et al., 2020), single employees were more satisfied than married one (Ghanayem et al., 2020).

In our study, we found salaries had a significant association with participation in decision making and training facilities. While low salary caused employees dissatisfaction, higher salary produced higher satisfaction (Ghanayem et al., 2020). Due to differences in salaries between groups, satisfaction of employees also significantly varies (Park et al., 2020). But in another study by Hossain et al. (2017), where they were measuring association between job satisfaction with work shifts and hours with work stress, revealed regarding pay and promotion no significance differences were found in job satisfaction among five work group.

This study revealed that demographic characteristics like religion, education and geographic location have no significant relation with any dependent variables. Job dissatisfaction is definitely not a good sign for any organization since it has an adverse effect on overall business. Working hours need to redesign. As organization's efficiency depends on employee's performance, it should be appraised from time to time. Proper training helps people to get rid of boredom. The organization can develop a participative environment to motivate employee which also give them a chance to take part in decision-making.

\section{LIMITATIONS OF THE STUDY}

Number of the participant was the major limitation. Few employees left without giving information may limit our study. Due to Covid -19 , we cannot give enough time in data collection. However, the study represented a clear picture of job satisfaction among private bank employees of Bangladesh.

\section{Conclusion And Recommendation}

This study revealed a significant inter-class relation between demographic variables like as gender, age, designation, salary, number of family member, marital status, working environment, service period and income of family with employees job satisfactory factors which were sharing in decision making, training, autonomy in work, gender discrimination, working hour, chance of promotion, increase knowledge \& capacity, and practice of MBO. However, the study showed that the overall level of employees work satisfaction was high.

To improve the condition some strategies could be developed based on the significant factors such as giving chance to employees to participate in decision making, fixing working hour, transparency, equitable and competitive compensation and promotion system, ensuring effective training and development program, removing gender discrimination.

\section{REFERENCES}

Agarwala, T. (2011). Strategic Human Resource Management (11th ed.). Oxford: New Delhi. https://india.oup.com/product/strategic-human-resource-management-9780195683592?

Ahmed, A. A. A. (2020). Corporate attributes and disclosure of accounting information: Evidence from the big five banks of China. J Public Affairs. e2244. https://doi.org/10.1002/pa.2244

Ali, T. \& Akhter, I. (2009). Job Satisfaction of Faculty Members in Private Universities -In Context of Bangladesh. International Business Research, 2(4), 167-175. https://doi.org/10.5539/ibr.v2n4p167 
Antoncic, J.A., \& Antoncic, B. (2011). Employee satisfaction, intrapreneurship and firm growth: a model, Industrial Management \& Data system, 111(4), 589-607. https://doi.org/10.1108/02635571111133560

Asadullah, A., Juhdi, N. B., Islam, M. N., Ahmed, A. A. A., \& Abdullah, A. (2019). The Effect of Reinforcement and Punishment on Employee Performance. ABC Journal of Advanced Research, 8(2), 47-58. https://doi.org/10.18034/abcjar.v8i2.87

Azad, M. R., Khan, W., \& Ahmed, A. A. A. (2011). HR Practices in Banking Sector on Perceived Employee Performance: A Case of Bangladesh. Eastern University Journal, 3(3), 30-39. https://doi.org/10.5281/zenodo.4043334

Bakotic, D. (2016). Relationship between job satisfaction and organizational performance, Economic Research-Ekonomska Istraživanja, 29:1, 118-130, https:/ / doi.org/10.1080/1331677X.2016.1163946

Balcazar, J. (2020). Religion, spirituality, and the workplace: A meta-analytic study on outcomes of job satisfaction, job performance, and organizational citizenship behaviors (Order No. 27834051). Available from ProQuest Dissertations \& Theses Global. (2410797912). Retrieved from https://search.proquest.com/docview/2410797912?accountid=35493

Bayona, J. A., Caballer, A., \& Peiró, J. M. (2020). The relationship between knowledge characteristics' fit and job satisfaction and job performance: The mediating role of work engagement. Sustainability, 12(6), 2336. https://dx.doi.org/10.3390/su12062336

Budría, S., \& Baleix, J. M. (2020). Offshoring, job satisfaction and job insecurity. Economics, 14(23), 1-32. http://dx.doi.org/10.5018/economics-ejournal.ja.2020-23

Buker, H. \& Dolu O. (2010). Police job satisfaction in Turkey: Effects of demographic, organizational and jurisdictional factors. International Journal of Comparatives and Applied Criminal Justice, 34(1): 25-51. https://doi.org/10.1080/01924036.2010.9678816

Chaulagain, N., \& Khadka, D. K. (2012). Factors Influencing Job Satisfaction among Healthcare Professionals at Tilganga Eye Centre, Kathmandu, Nepal. International Journal of Scientific \& Technology Research, 1(11), 32-36. http://www.ijstr.org/paper-references.php?ref=IJSTR-1212$\underline{5543}$

Clark, A. E. (1997). Job satisfaction and gender: Why are women so happy at work? Labour economics, 4(4), 341-372. https://doi.org/10.1016/S0927-5371(97)00010-9

Evans, J. R., \& Jack, E. P. (2003). Validating key results linkages in the Baldrige Performance Excellence Model. The Quality Management Journal, 10, 7-24. https://doi.org/10.1080/10686967.2003.11919060

Falkenburg, K. and Schyns, B. (2007). Work satisfaction, organizational commitment and withdrawal behaviors, Management Research News, 30(10), 708-723. https://doi.org/10.1108/01409170710823430

Faragher, E. B., Cass, M., \& Cooper, C. L. (2005). The relationship between job satisfaction and health: a meta-analysis. Occup Environ Med. 2005 Feb; 62(2):105-12. https://doi.org/10.1136/oem.2002.006734

Farooqui, M. S. \& Nagendra, A. (2014). The Impact of Person organization Fit on Job Satisfaction and Performance of the Employees. Procedia Economics and Finance, 11, 122 - 129. https://doi.org/10.1016/S2212-5671(14)00182-8

Farrell, D., \& Stamm, C. L. (1988). Meta-analysis of the correlates of employee absence. Human Relations, 41(3), 211-227. https://doi.org/10.1177\%2F001872678804100302

Ghanayem, M., Srulovici, E., \& Zlotnick, C. (2020). Occupational strain and job satisfaction: The job demand-resource moderation-mediation model in haemodialysis units. Journal of Nursing Management, 28(3), 664-672. http://dx.doi.org/10.1111/jonm.12973

Hakim, M. A. (2015). Organizational Culture and Job Satisfaction in Bank: Perceptions and Reactions of Employees. Global Disclosure of Economics and Business, 4(2), 167-180. https://doi.org/10.18034/gdeb.v4i2.145 
Haque, A. B., \& Ahmed, A. A. (2016). Striving for Work-Life Balance at Entry Level Jobs: Challenges to Develop Professionalism in Bangladesh. Asian Business Review, 6(2), 97-104. https://doi.org/10.18034/abr.v6i2.33

Hassan, M., Azmat, U., Sarwar, S., Adil, I. H., \& Gillani, S. H. M. (2020). Impact of Job Satisfaction, Job Stress and Motivation on Job Performance: A Case from Private Universities of Karachi. Kuwait Chapter of the Arabian Journal of Business and Management Review, 9(2), 31-41. Retrieved from https://search.proquest.com/docview $/ 2434244417$ ? accountid=35493

Heidarian, A. R., Kelarijani, S. E. J., Jamshidi, R., \& Khorshidi, M. (2015). The relationship between demographic characteristics and motivational factors in the employees of social security hospitals in Mazandaran. Caspian J Intern Med, 6(3), 170-174. https://pubmed.ncbi.nlm.nih.gov/26644886/

Herzberg, F., Mausner, B., \& Snyderman, B. B. (1959). The motivation to work. John Wiley and Sons. https://trove.nla.gov.au/work/9786532

Hoboubi, N., Choobineh, A., Ghanavati F. K., Keshavarzi, S., \& Hosseini, A.A. (2017). The Impact of Job Stress and Job Satisfaction on Workforce Productivity in an Iranian Petrochemical Industry. Saf Health Work; 8(1), 67-71. https://doi.org/10.1016/j.shaw.2016.07.002

Hossain, M. A., Mustafi, M. A. A., Islam, M. M., \& Islam, M. R. (2017). Organizational Environment and Nurses' Job Satisfaction: A Study on Private Hospital in Bangladesh. American Journal of Trade and Policy, 4(1), 25-32. https://doi.org/10.18034/aitp.v4i1.413

Huda, S. S. M. S., Akhtar, N., \& Akhtar, A. (2011). Employee's View on Job Satisfaction: A Study on Garments Industry in Bangladesh. Indus Journal of Management \& Social Sciences, 5(1), 1-9. https://ideas.repec.org/a/iih/journl/v5y2011i1p1-9.html

Huning, T. M., Hurt, K. J., \& Frieder, R. E. (2020). The effect of servant leadership, perceived organizational support, job satisfaction and job embeddedness on turnover intentions. Evidence Based HRM, 8(2), 177-194. http://dx.doi.org/10.1108/EBHRM-06-2019-0049

Ireri, K. (2016). High Job satisfaction despite low income: A national study of Kenyan journalists. $\begin{array}{lllll}\text { Journalism } \mathcal{E} \text { Mass Communication } & \text { Quarterly, 93(1), 164-186. }\end{array}$ https://doi.org/10.1177\%2F1077699015607334

Islam, M. R., Rasul, M. T., Ullah, G. M. W. (2012). Analysis of the Factors that Affect Job Satisfaction: A Case Study on Private Companies Employees of Bangladesh. European Journal of Business and Management, 4(4), 35-47. https:/ /iiste.org/Journals/index.php/EJBM/article/view/1429

Islam, S. M. S. (2016). A New Method to Measure the Job Satisfaction Level of an Employee. Engineering International, 4(1), 19-24. https://doi.org/10.18034/ei.v4i1.182

James, G. (2020). Relationship between intrinsic job satisfaction, extrinsic job satisfaction, and turnover intentions in luxury hotels (Order No. 27739368). Available from ProQuest Dissertations \& Theses Global. (2378077937). Retrieved from https://search.proquest.com/docview/2378077937?accountid=35493

Khan, S. S. (2015). Measuring Job Satisfaction Level of Employees using Demographics: A Study of HDFC Bank. Global Journal of Management and Business Research: An Administration and Management, 15(2), 38-48. https:/ / globaljournals.org/GJMBR_Volume15/6-Measuring-Job-Satisfaction.pdf

Kumar, D. (2016). Job Satisfaction of Commercial Bank Employees in Bangladesh: An Empirical Study. ABC Journal of Advanced Research, 5(2), 61-70. https://doi.org/10.18034/abcjar.v5i2.59

Molla, M. I. H. (2015). Ensuring Job Satisfaction for Managing People at Work. Global Disclosure of Economics and Business, 4(2), 155-166. https://doi.org/10.18034/gdeb.v4i2.144

Moortezagholli, Z. (2020). Understanding the job satisfaction and retention of lived experiences of millennial healthcare leaders and their perspectives about job satisfaction and retention (Order No. 27956279). Available from ProQuest Dissertations \& Theses Global. (2404053999). Retrieved from https://search.proquest.com/docview/2404053999?accountid=35493 
Park, J., Ahn, J., Han, S., Back, K., \& An, M. (2020). Exploring internal benefits of medical tourism facilitators' satisfaction: Customer orientation, job satisfaction, and work performance. Journal of Healthcare Management / American College of Healthcare Executives, 65(2), 90-105. http://dx.doi.org/10.1097/JHM-D-18-00168

Popoola, S. O. (2009). Organizational commitment of records management personnel in Nigerian private universities, Records Management Journal, 19(3): $204 \quad-217$. https://dx.doi.org/10.1108/09565690910999193

Rahman, M., Ashraf, A., Hasan, N., Hoshen, S., Chowdhury, R. H. K., \& Moni, M. (2017). Job Satisfaction Levels among Employees of Private Commercial Banks at Chuadanga District in Bangladesh. J Bus Fin Aff, 6(2), 1-8. https://doi.org/10.4172/2167-0234.1000256

Rahman, S. M. (2020). Relationship between Job Satisfaction and Turnover Intention: Evidence from Bangladesh. Asian Business Review, 10(2), 99-108. https://doi.org/10.18034/abr.v10i2.470

Shaikh, S.H., Shaikh, H., \& Shaikh, S. (2019). The Impact of Job Satisfaction and Job Dissatisfaction on Herzberg Theory: A Case Study of Meezan Bank Limited and National Bank Limited. International Journal of Business and Social Science. 10(6), 143-147. https://doi.org/10.30845/ijbss.v10n6p16

Talukder, S., Talukder, F. H., \& Alam, J. (2014). Job Dissatisfaction and Turnover: Bangladesh Perspective. European Journal of Business and Management, 6(17), 117-125. https://www.iiste.org/Journals/index.php/EJBM/article/view/13576

Vickovic, S. G., \& Morrow, W. J. (2020). Examining the influence of Work-Family conflict on job stress, job satisfaction, and organizational commitment among correctional officers. Criminal Justice Review, 45(1), 5-25. http://dx.doi.org/10.1177/0734016819863099

Weerasinghe, I.M.S., Senavirathna, C.J. \& Dedunu, H.H. (2017). Factors Affecting to Job Satisfaction of Banking Employees in Sri Lanka Special Reference Public and Private Banks in Anuradhapura District. Business and Management Horizons, 5(1), 62-73. https://ssrn.com/abstract=2978608

Yamane, T. (1967) Statistics an Introductory Analysis. 2nd Edition, New York, Harper and Row. https://www.gbv.de/dms/zbw/252560191.pdf 


\section{How to Cite this Article}

Hasan, M. N., Afrin, S., Rahman, M., \& Ahmed, A. A. A. (2020). Assessing the Nature of Job Satisfaction Level: A Study on Private Bank Employees in Bangladesh. Global Disclosure of Economics and Business, 9(2), 67-88. https://doi.org/10.18034/gdeb.v9i2.511 